\title{
Using Atomic Force Microscopy to Measure Anti-Adhesion Effects on Uropathogenic Bacteria, Observed in Urine after Cranberry Juice Consumption
}

\author{
Laila Abu-Lail $^{1}$, Yuanyuan Tao ${ }^{1}$, Paola A. Pinzón-Arango ${ }^{1}$, Amy Howell ${ }^{2}$, Terri A. Camesano ${ }^{1 *}$ \\ ${ }^{1}$ Department of Chemical Engineering, Worcester Polytechnic Institute, Worcester, USA; ${ }^{2}$ School of Environmental and Biological \\ Sciences, Rutgers University, Newark, USA. \\ Email: "terric@wpi.edu
}

Received August $16^{\text {th }}, 2012$; revised September $18^{\text {th }}, 2012$; accepted September $30^{\text {th }}, 2012$

\begin{abstract}
A volunteer was given cranberry juice cocktail (CJC) or water to drink, and urine was collected at 2 and 8 hours after consumption, in order to quantitatively determine whether adhesion forces were changed for the volunteer after CJC consumption. Atomic force microscopy (AFM) was used to measure adhesion forces between bacteria and a silicon nitride tip. Forces between Escherichia coli or Staphylococcus aureus and the AFM tip were lower in the urine after the volunteer consumed CJC, compared to drinking water. A steric model was applied to the AFM data, in order to quantify how the urine changed the properties of the bacterial surfaces. There was a small decrease in the equilibrium length of surface molecules on the bacteria when in the post-CJC urine, compared to the post-water urine. However, these changes were not statistically significant. We hypothesize that post-CJC urine imparts subtle changes on the molecules of the bacterial surfaces, and that these changes lead to the reduction in adhesion with the AFM probe.
\end{abstract}

Keywords: Vacciniumm macrocarpon; Urinary Tract Infections; E. coli; S. aureus; AFM

\section{Introduction}

Considered to be one of the most common bacterial infections, urinary tract infections will affect almost half of all women in their lifetime, and nearly 1 in 3 women will have a UTI requiring antibiotics before age 24 [1]. Alternative therapies for UTIs have drawn increasing interest due to concern of antibiotic resistant bacteria. The American cranberry (Vacciniumm macrocarpon) is a potential therapeutic for UTIs, and several clinical studies have shown that consumption of cranberry can help prevent UTIs or recurrences of UTIs among elderly people [2,3], children [4], and sexually active women with previous UTIs [5], although it has been difficult to demonstrate longer term benefits of cranberry as a means to prevent UTI recurrence over long times [6]. Cranberry products are consumed by many healthy women as a preventive measure, but further research is needed to elucidate how oral consumption of cranberry affects the activity of uropathogenic bacteria, and to better understand the type of cases where cranberry can be effective for UTI prevention. The timing and dose needed for this thearapeutic effect are also being investigated [7].

\footnotetext{
${ }^{*}$ Corresponding author.
}

E. coli spp. are by far the most predominant species that cause UTIs, accounting for $70 \%-95 \%$ of all UTIs. In addition, P-fimbriated E. coli strains are the primary cause for $90 \%$ of acute pyelonephitis cases [8]. Remaining UTIs are caused by various organisms, including staphylococci, proteus organisms, Klebsiella spp., and Enterococcus faecalis. The incidence of UTIs related to $S$. aureus was found to be $3.5 \%$, based on an analysis of 321 patients over a 3 -year period [9]. Pathogenic bacteria have the ability to adhere to inert surfaces, such as a catheter, which leads to device-associated infections. Catheter-related UTIs tend to be caused by Gramnegative bacilli [10], including pseudomonads [11], fungi, and Gram-positive cocci, which includes staphylococci $[10,12,13]$.

The first step in the initiation of a UTI, or any bacterial infection, is that bacteria must attach to a surface, such as a catheter placed inside the body or the epithelial cells that line the urinary tract. The attachment of bacteria to abiotic materials is a complicated process that is affected by various physicochemical properties of both bacterial cells and substratum surface. Understanding the role of cranberry juice components on interactions within this process is critical in developing novel strategies for the 
efficient control of bacterial attachment and biofilm formation.

Exposure to cranberry juice has been shown to decrease bacterial attachment, adhesion, and biofilm formation. For example, cranberry juice inhibited the attachment of E. coli to human uroepithelial cells, red blood cells, and resin beads coated with receptors that bind to P-fimbriae, the molecules on uropathogenic E. coli cell surfaces that facilitate adhesion to uroepithelial cells [14-16] In an attempt to gain more quantitative information on how cranberry juice affects bacterial adhesion, adhesion force measurements have been made using atomic force microscopy (AFM). AFM experiments demonstrated that P-fimbriated bacteria alter the conformation of their fimbriae in the presence of cranberry juice cocktail (CJC) [17], and adhesion forces between $E$. coli and an AFM silicon nitride tip were reduced when bacteria were grown in the presence of light CJC or in proanthocyanidins extracted from the same juice [14]. In addition to studies with juice, the urine of volunteers who have consumed CJC has been investigated to determine if anti-adhesive or anti-biofilm compounds remain in urine. We previously reported that AFM adhesion force measurements between uropathogenic $E$. coli were decreased in the presence of urine from a volunteer who had consumed CJC [18]. In addition, DiMartino et al. showed that cranberry juice consumption decreased biofilm development of uropathogenic $E$. coli in the urine compared with placebo [19,20].

In recent years, AFM has emerged as a valuable and powerful tool for understanding the nanoscale properties of living cells. A major advantage of AFM is that it allows measurements of the forces associated with single biomolecules in aqueous media. This is ideal for biological applications, where AFM can be used to investigate the individual bacterial cells in buffer solution, without the need of fixing, staining, or labeling. AFM has been extremely useful for providing high-resolution topographic images of cell surfaces and biomolecules [21-23]. In addition, quantitative information on bacterial interaction forces and adhesion can be provided though analysis of AFM data [24]. Furthermore, in order to quantify AFM data and use it to interpret behavior of bacterial surfaces, a steric model can be applied to force vs. distance profiles $[25,26]$. This model allows us to characterize the physicochemical properties of polymers on a bacterial surface.

In this work, AFM was used to investigate the interacttions between bacteria ( $S$. aureus or $E$. coli) and a model surface (silicon nitride tip) in the presence of urine samples containing cranberry juice components. We focused on representative bacterial pathogens in this study, namely, two types of clinical $E$. coli that are associated with UTIs (strains BF1023 and CFT073, both of which have P-fimbriae), and $S$. aureus ATCC43866. While $E$. coli is the most common uropathogen, $S$. aureus is a pathogen frequently involved in device-centered infections, has the ability to adhere to catheters and other medical devices and form biofilm [13], and is a growing concern due to multi-drug antibiotic resistance. The steric model was applied so that changes in the bacterial surfaces due to exposure to the urine of a volunteer who had consumed CJC could be quantified. To our knowledge this is the first study to characterize the effect of CJC byproducts in urine on the adhesion properties of Staphylococcus aureus (S. aureus) using AFM.

\section{Materials and Methods}

\subsection{Urine Samples}

De-identified urine samples were sent to Worcester Polytechnic Institute (WPI) from Rutgers University. An arbitrary, crossover design was applied, with a single volunteer. The volunteer was a healthy Caucasian male, aged 42 . He drank 16 oz. (240 mL) of commercial cranberry juice cocktail (CJC) or water. After consuming the single dose, urine was collected at two time intervals (2 and 8 hours). Samples were immediately frozen and shipped to WPI, where they were stored at $-20^{\circ} \mathrm{C}$. Urine samples were later thawed and filtered though $0.8 \mu \mathrm{m}$ (Pall Corp., East Hills, NY) and $0.2 \mu \mathrm{m}$ (VWR International $^{\mathrm{TM}}$, West Chester, PA) polyethersulfone syringe filters, sequentially.

\subsection{Bacteria Cultures}

Two E. coli clinical strains that cause acute pyelonephitis (AP) or cystitis, and one $S$. aureus strain (ATCC 43866) were selected as model bacteria. All bacteria were purchased from ATCC (Manassas, VA). The two E. coli strains were; E. coli CFT073 and E. coli BF1023, both are P-fimbriated strains from different classes. CFT073 expresses P-fimbriae from class II and was isolated from the blood and urine of a woman with AP, and BF1023 expresses P-fimbria from classes I and III and was isolated from a female patient with cystitis.

E. coli strains were cultured at $37^{\circ} \mathrm{C}$ in colonizing factor antigen (CFA) media. CFA media is composed of $1 \%$ $(\mathrm{w} / \mathrm{v})$ casamino acids (Bacto $^{\mathrm{TM}}$, Sparks, MD 21152), $0.078 \%(\mathrm{w} / \mathrm{v})$ yeast extract (Bacto ${ }^{\mathrm{TM}}$, Sparks, MD 21152), $0.4 \mathrm{mM} \mathrm{M \textrm {SO } _ { 4 }}$ (Sigma-Aldrich), $0.04 \mathrm{mM} \mathrm{MnCl}_{2}$ (Sigma-Aldrich) in ultrapure water, and the $\mathrm{pH}$ was adjusted to 7.4 using sodium hydroxide (Sigma-Aldrich). For culture plates, $2 \%$ agar (Bacto $^{\mathrm{TM}}$, Sparks, MD 21152) was added to the media. $S$. aureus cultures were grown overnight in Luria-Bertani (LB) medium at $37^{\circ} \mathrm{C}$. Bacte- 
ria were harvested at late exponential phase, when the absorbance of the culture was $0.9-1.1$ at a wavelength of $600 \mathrm{~nm}$, measured with a spectrophotometer (Thermo Spectronic, Rochester, NY).

\subsection{Bacterial Cell Preparation for AFM}

Bacteria were immobilized to acid-cleaned glass slides using a cross-linking reaction between amine and carboxyl groups [18]. The cleaned glass slides were immersed in 10\% 3-aminopropyltrimethoxysilane in methanol for $20 \mathrm{~min}$. Glass slides were rinsed with methanol and ultrapure water. Bacterial cultures were washed thee times with ultrapure water by centrifuging at $7000 \mathrm{rpm}$ for $10 \mathrm{~min}$, removing the supernatant, and resuspending the bacterial pellets in ultrapure water. A mixture of 300 $\mu \mathrm{l}$ of $100 \mathrm{mM}$ EDC (1-ethyl-3-(3-dimethylaminopropyl) carbodiimide hydrochloride) and $10 \mathrm{ml}$ of the bacterial solution was prepared and incubated for 10 minutes at $37^{\circ} \mathrm{C}$ with rotation at $18 \mathrm{rpm}$. After $10 \mathrm{~min}$, a $600 \mu \mathrm{l}$ aliquot of $40 \mathrm{mM}$ NHS (N-hydroxysulfosuccinimide) solution was added to the bacterial solution and incubated at $37^{\circ} \mathrm{C}$ for $10 \mathrm{~min}$ with rotation at $18 \mathrm{rpm}$. The final solution was then added to the aminosilane-treated glass slides and agitated at $70 \mathrm{rpm}$ for $4 \mathrm{~h}$ to allow bacteria to bind to the glass slides. Previous work has shown that the viability and morphology of bacterial cells is not affected by the binding process [27].

\subsection{AFM Force Measurements}

AFM interaction forces were measured using an MFP3D-BIO AFM (Asylum Research, Santa Barbara, CA). Silicon nitride cantilevers were used, with an average spring constant of $0.12 \pm 0.02 \mathrm{~N} / \mathrm{m}$ (DNPS, Veeco Metrology) calibrated and estimated from the power spectral density of the thermal noise fluctuations in air. The normal photodiode sensitivity was determined in liquid by measuring the slope of the constant compliance region of the force curve on a hard surface. Before experiments, the cantilevers were exposed to ultraviolet light for $1 \mathrm{~h}$ to remove any potential organic contamination. All force measurements were performed with the bacterial-coated slides and cantilevers immersed in the urine sample. During this time, we found that the bacterial cells remain viable but are not actively growing. For each condition studied, at least five individual bacterial cells were probed, and ten force measurements were recorded in the center of each bacterium.

\subsection{Steric Model}

The repulsive interactions between the bacterium and AFM tip were fit using a model for steric interactions.
The steric model describes the forces between interacting surfaces that have thermally mobile surface groups [28]. A common type of thermally-diffuse surface is a surface covered with chain molecules that dangle out into the solution, such as polymers. When another surface is approaching these dangling chains in a well-defined volume, a repulsive force known as "steric" force arises. The steric model originally developed by Alexander [29] and de Gennes [28] (AdG) to describe the forces between two flat surfaces with high coverage of grafted or adsorbed polymers. In addition, the AdG model has been successfully applied to the characterization of bacterial polymers $[24,26]$ and to quantify interactions between a polymer-covered surface, such as hyaluronic acid [30], with model surfaces. Adaption of the AdG model to account for the spherical AFM probe interacting with a flat surface was performed [25], where the steric model takes the following form

$$
F(D)=\frac{8 k T R \pi L_{o}}{35 s^{3}}\left[7\left(\frac{L_{o}}{d+\delta}\right)^{5 / 4}+5\left(\frac{d+\delta}{L_{o}}\right)^{7 / 4}-12\right],
$$

where $F$ is the steric repulsive force measured by AFM, as a function of the separation distance $(D), k$ is the Boltzmann constant, $T$ is the absolute temperature, $R$ is the tip radius, $\mathrm{s}$ is the average distance between grafting sites, and $L_{o}$ is the equilibrium length of the polymer layer. The separation distance, $D$, was used as the sum of the measured distance $(d)$ and the offset distance $(\delta)$, according to the procedure of Chang et al. [30]. Although modeling has sometimes been done without this parameter, the inclusion of $\delta$ offset enables improved fitting because it provides an estimate for the layer thickness at the maximal applied force. By fitting the steric model to the approach portions of the AFM force cycles, the equilibrium polymer length, spacing, and offset distance were calculated. For each bacterium and condition studied, average values of the equilibrium polymer length and spacing were reported in plots, but individual values (with no averaging) of these parameters were statistically compared. At each time point, the two way ANOVA test was used to compare the data sets from the two treatments (water or $\mathrm{CJC}$ ).

\subsection{Adhesion Force Analysis}

Each AFM force cycle has two parts, the approach of the AFM tip to the bacterium, and the retraction of the tip away from the bacterial sample. During the approach portion of the force cycle, repulsive steric forces are often observed, as described. However, the retraction portion of the cycle is useful to analyze because this is where adhesion forces between the AFM tip and molecules protruding from the bacterial surface can be ob- 
served. For each experimental condition studied, we collected the data from all adhesion events and calculated an average adhesion force. Both individual (representa- tive) retraction force profiles are presented, along with averaged adhesion forces for each experimental condi- tion. Statistical analyses using SigmaStat ${ }^{\circledR} 2.03$ (Systat Software, San Jose, CA) were also performed The two way ANOVA test was used to compare the two groups that were treated with urine samples collected after ( 2 hours and 8 hours) of water or CJC consumption.

\section{Results and Discussion}

\subsection{Analysis of Adhesion Forces}

The retraction portions of the AFM force cycles were examined to gain information regarding the adhesion forces between biomolecules on the bacterial surface and AFM tip. Individual adhesion forces from 50 retraction curves were combined and used to calculate an average adhesion force between the bacterial cell surface and AFM tip. Figure 1 shows the average adhesion forces for each bacterial strain in the urine collected after CJC consumption (post-CJC urine) and after water consumption (post-water urine), at two time intervals ( 2 and $8 \mathrm{~h}$ ) following beverage consumption. In addition, adhesion forces for different treatments and at different time points were compared statistically to obtain more reliable results (Figure 1). Tested data groups were considered significantly different if the resulting P-value was less than 0.05 . For all bacterial strains, the adhesive forces between bacterial cells and AFM tip in the post-CJC urine were significantly less than the adhesive forces between bacterial cells and AFM in the post-water urine (Figure 1). E. coli strains demonstrated a significant decrease in the adhesive forces with time after CJC consumption and showed no significant changes in the adhesive forces with time after water consumption. Strain BF1023 showed a greater reduction in the adhesive forces with time after CJC consumption than CFT073. For BF1023, the average adhesive forces decreased from $(0.86 \pm 0.05 \mathrm{nN})$ at $2 \mathrm{~h}$ to $(0.29 \pm 0.01 \mathrm{nN})$ at $8 \mathrm{~h}$ following CJC consumption, while CFT073 adhesive forces decreased from $(0.77 \pm 0.04 \mathrm{nN})$ at $2 \mathrm{~h}$ to $(0.38 \pm 0.02$ $\mathrm{nN})$ at $8 \mathrm{~h}$ following CJC consumption. On the other hand, adhesion forces between $S$. aureus and the AFM tip at $2 \mathrm{~h}(0.124 \pm 0.01 \mathrm{nN})$ and $8 \mathrm{~h}(0.115 \pm 0.01 \mathrm{nN})$ after CJC consumption were not statistically different from one another. Similarly, the adhesive forces between S. aureus and the AFM tip at $2 \mathrm{~h}(0.24 \pm 0.03 \mathrm{nN})$ and 8 h $(0.21 \pm 0.02 \mathrm{nN})$ after water consumption were not statistically different from one another. Comparing all strains under various conditions, BF1023 had the highest adhesion forces with the AFM tip and S. aureus had the

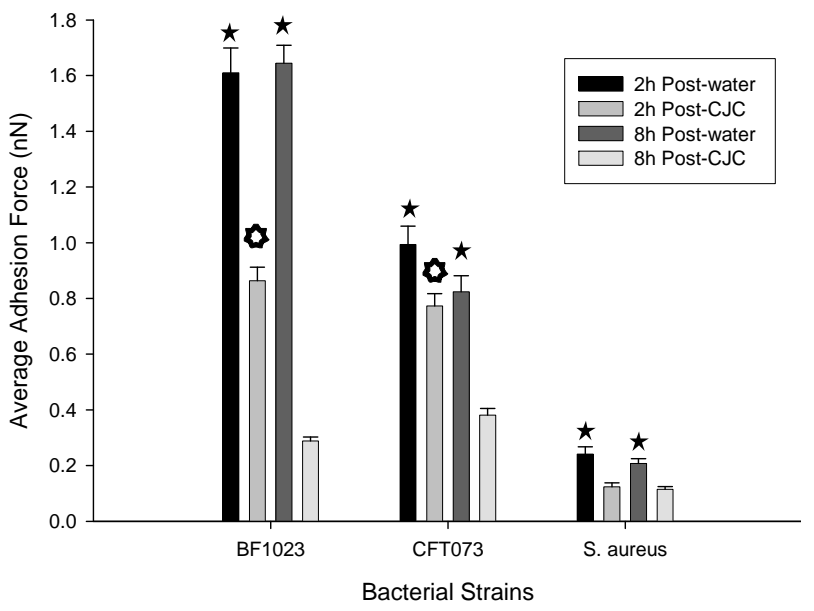

Figure 1. Average adhesion forces for E. coli BF1023, E. coli CFT073, and $S$. aureus after $2 \mathrm{~h}$ and $8 \mathrm{~h}$ of beverage consumption (water or CJC). Error bar indicates the standard error of the mean. 5-point black star indicates a statistically significant difference between two treatments at one time point. 7-point black star indicates a statistically significant difference between two times at one treatment condition.

weakest adhesion forces with the AFM tip. For example, at $2 \mathrm{~h}$ after water consumptions, the adhesion forces between the AFM tip and BF1023, CFT073, and S. aureus were $(1.61 \pm 0.09),(0.99 \pm 0.07)$, and $(0.24 \pm 0.03)$, respectively. The silicon nitride AFM tip has a weakly negative electrostatic charge in solution. It is not meant to represent a catheter or an epithelial cell exactly, but can be used as a model probe to screen for non-specific physicochemical interactions between the bacterium and the probe material. It can also be used to help rank the strength of different samples or treatments.

In all the strains tested, there was a significant decrease in the adhesion forces between bacteria and AFM tip in the post-CJC urine as compared to the post-water urine both at 2 and $8 \mathrm{~h}$ after beverage consumption. These results confirm the existence of anti-adhesive molecules in the urine after oral consumption of CJC. This trend is also easily visualized by examining representative adhesion force profiles for the $8 \mathrm{~h}$ conditions (Figure 2). All 3 bacterial strains tested show an obvious decrease in the magnitude of the adhesion forces, as well as the distance at which those force extend, in the presence of post-CJC urine at $8 \mathrm{~h}$, compared to post-water urine at $8 \mathrm{~h}$. A similar trend in the data was observed for the $2 \mathrm{~h}$ post condition (data not shown), in which adhesion forces were lower and extended over shorter distances in post-CJC urine than in post-water urine.

However, the effect of time post-consumption on the adhesion forces differed among the strains. Within two hours of drinking CJC, S. aureus had the highest reducetion in the adhesion forces between the post-CJC urine 


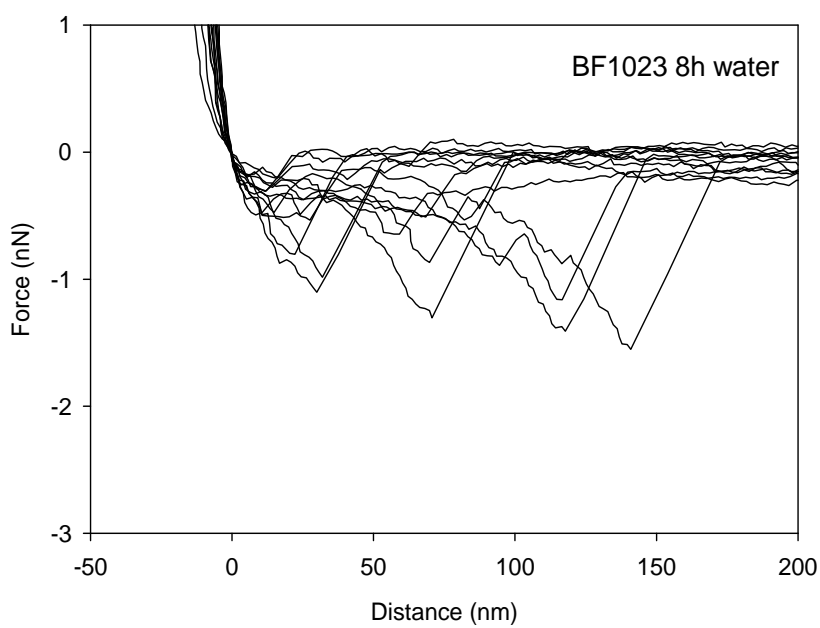

(a)

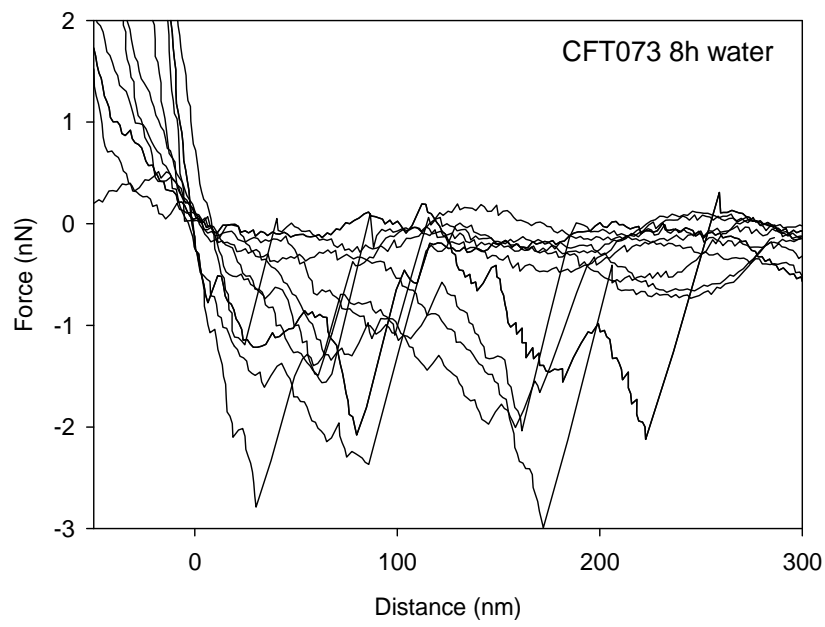

(c)

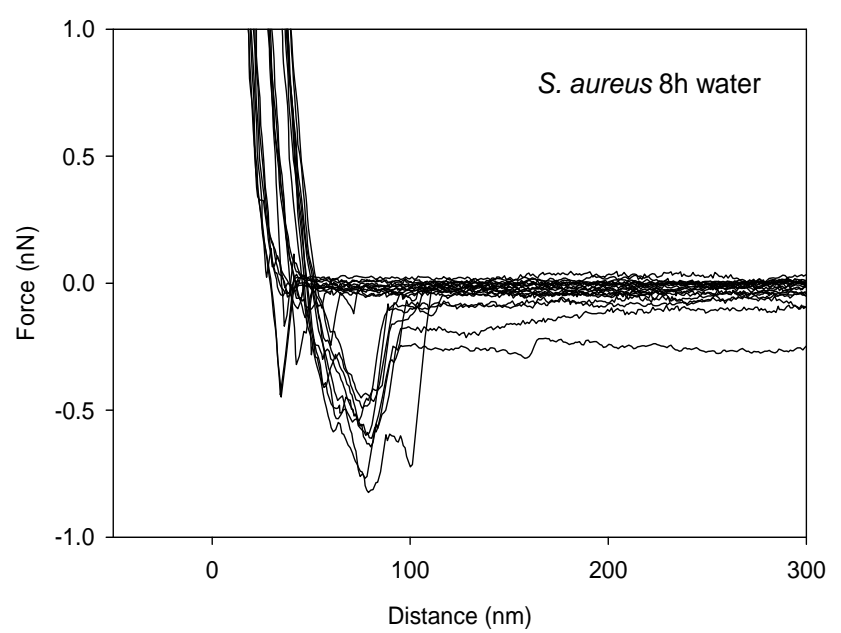

(e)

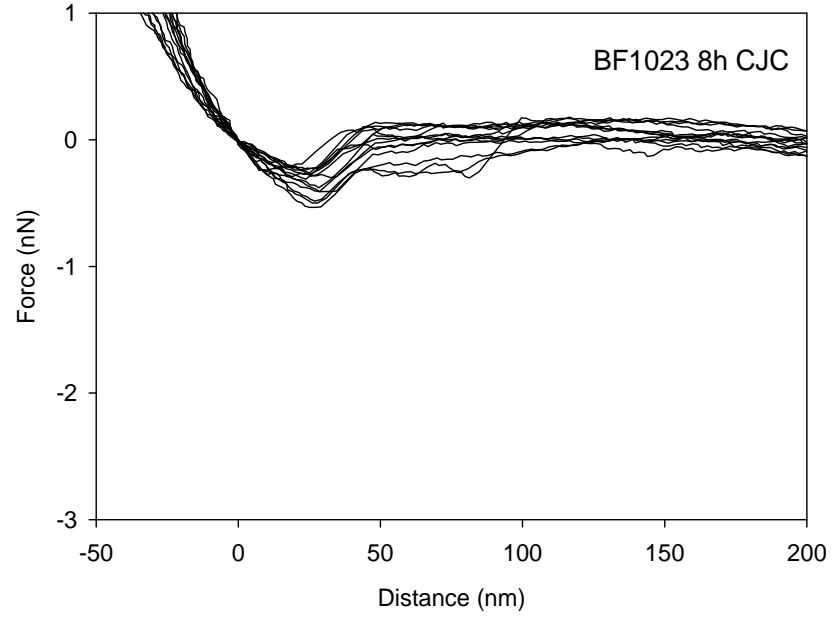

(b)

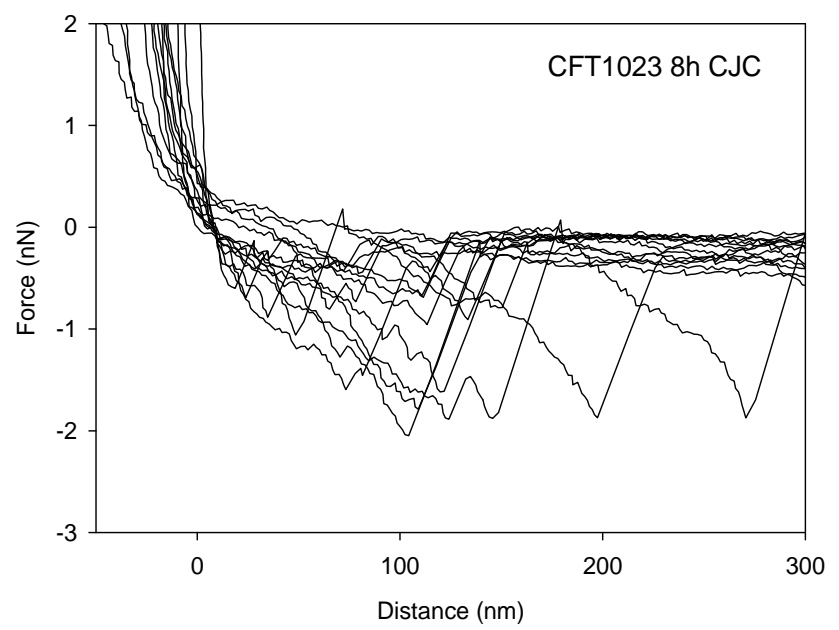

(d)

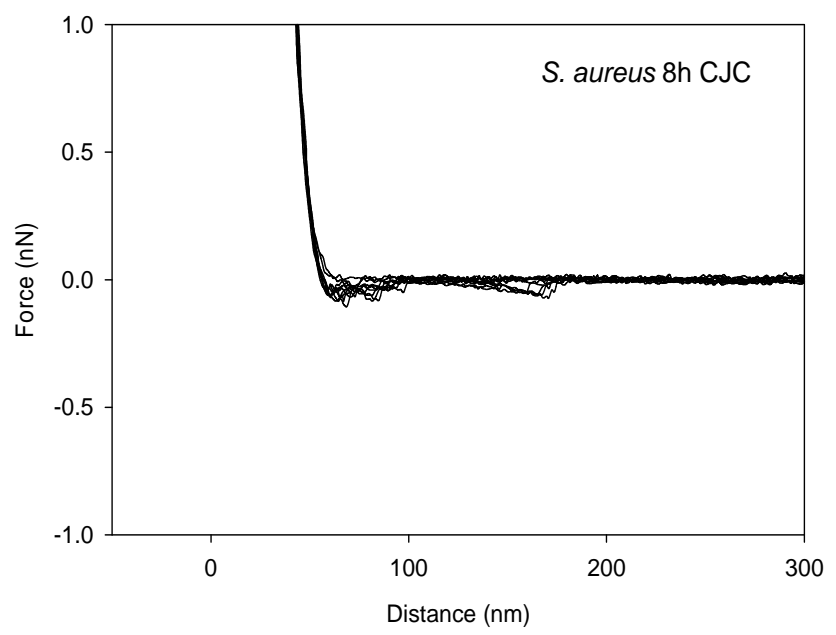

(f)

Figure 2. Representative adhesion force data corresponding to 8 hours after consumption of water or CJC for all thee bacterial strains. In all cases, the right panels (b), (d), and (f) show much lower adhesion forces than the left panels (a), (c), and (e), which correspond to CJC and water, respectively. Similar types of data trends were observed 2 h post-beverage consumption (data not shown). For each condition, 50 force curves were collected. For clarity, only representative plots are displayed. 
and post-water urine, followed by E. coli $\mathrm{BF} 1023$ and finally by $E$. coli CFT 073 (Figure 1). However, after 8 hs of beverage consumption, the scenario was different, E. coli $\mathrm{BF} 1023$ had the highest reduction in the adhesion forces between the post-CJC urine and post-water urine followed by E. coli CFT073 and then by S. aureus. By considering changes in the post-CJC urine data as a function of time, our results demonstrated that the adhesion forces for the two E.coli strains after drinking CJC continued to decrease over the eight hour measurement period while the adhesion forces of $S$. aureus appeared to reach a plateau 2 hs post-CJC. Furthermore, the adhesive forces between AFM tip and $S$. aureus were lower than the adhesive forces between E. coli strains and the AFM tip.

The differences in how E. coli and S. aureus, and even among the two $E$. coli strains, reacted to post-CJC urine will also depend in part on the nature of the bacterial surfaces. E. coli CFT073 and BF1023 each exhibit mannose resistant hemagglutination. CFT073 expresses Pfimbriae, while the adhesins on BF1023 have not been characterized. S. aureus has surface protein adhesins that are smaller than E. coli fimbriae [31]. There are no published reports in which AFM has been used to characterize how $S$. aureus is affected by cranberry juice or its byproducts. However, a recent study found that cranberry extracts could inhibit growth and biofilm formation for $S$. aureus and other Staphylococcus spp., as well as inhibiting biofilm formation (but not growth), for E. coli [32]. The difference in activity of cranberry extracts against Gram-negative and Gram-positive bacteria was suggested by LaPlante et al. to be due to the lack of outermembrane lipopolysaccharides for the Gram-positive bacteria, which could then make it easier for cranberry or other antimicrobial compounds to penetrate the cell membrane.

\subsection{Bacteria Surface Characterization and Steric Model Application}

Data from all 50 force curves were used to determine the parameters of the steric model, the equilibrium length, the spacing between polymer units, and the offset. For strain $S$. aureus, the correlation coefficient values obtained by fitting the steric model to 50 approach curves were higher than $0.98\left(\mathrm{R}^{2}>0.98\right)$, while for strains CFT073 and BF1023, the correlation coefficient values were $\left(R^{2}>0.95\right)$ and $\left(R^{2}>0.90\right)$, respectively.

For each bacterial strain, the two way ANOVA test was applied to the steric model parameters for two time intervals $(2$ and $8 \mathrm{~h}$ ) and at two treatments (post-water and post-CJC). A given set of two tested groups was considered significantly different if the resulting P-value was less than 0.05 . Figure 3 shows the average equilibrium length for each given strain as a function of time and treatment after beverage consumption. For S. aureus, our statistical analysis demonstrated that there was a significant difference between the equilibrium lengths in the urine collected $2 \mathrm{~h}$ after water consumption and the urine collected $2 \mathrm{~h}$ after CJC consumption. The equilibrium polymer length in the urine $2 \mathrm{~h}$ post-CJC consumption was $60 \%$ less than its equivalent value in urine $2 \mathrm{~h}$ post-water consumption. However, the decrease in the equilibrium length did not continue with time, since the post-water urine and the post-CJC urine groups showed no significant difference in their average equilibrium lengths after $8 \mathrm{~h}$ of beverage consumption.

Neither E. coli strains, BF1023 nor CFT073, showed a significant change in the average equilibrium length between the post-water urine group and the post-CJC urine group during the $2 \mathrm{~h}$ or the $8 \mathrm{~h}$ following beverage consumption. In general, $S$. aureus average equilibrium polymer lengths were shorter than the average equilibrium polymer lengths of $E$. coli strains BF 1023 and CFT073. The average equilibrium polymer lengths of strain S. aureus were in the range of $48-116 \mathrm{~nm}$ and the average equilibrium polymer lengths of strains BF1023 and CFT073 were in the range of $119-205 \mathrm{~nm}$ and 73 $420 \mathrm{~nm}$, respectively.

Another parameter obtained from fitting the steric model to the AFM approach curves is the spacing between polymers sites, which can represent how densely the bacterial surface is covered with interacting molecules (Figure 4). In all cases, S. aureus had smaller average spacing $(2.0-3.1 \mathrm{~nm})$ compared to the average spac-

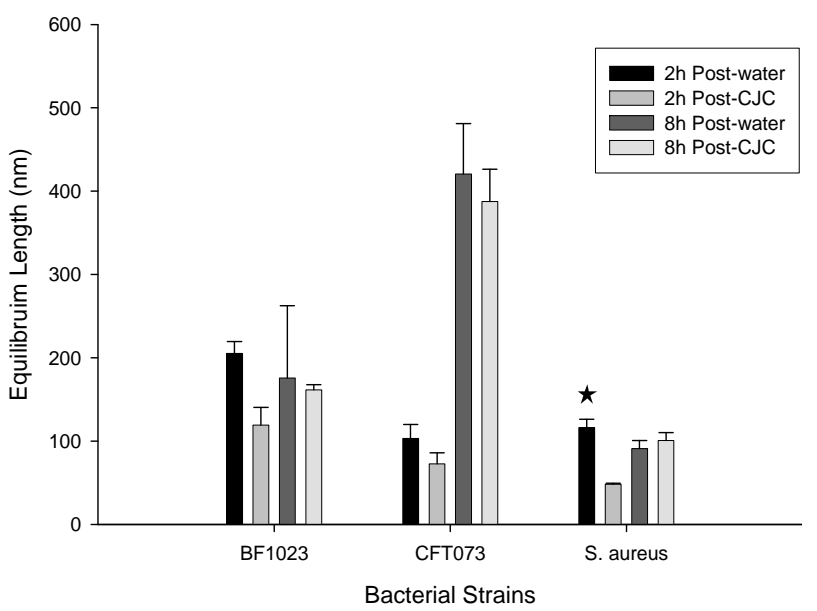

Figure 3. Average equilibrium lengths of $E$. coli BF1023, $E$. coli CFT073, and $S$. aureus at two time intervals ( 2 and $8 \mathrm{~h}$ ) after beverage consumption (water or CJC). Error bar indicates the standard error of the mean. 5-point black star indicates a statistically significant difference between two treatments at one time point. 


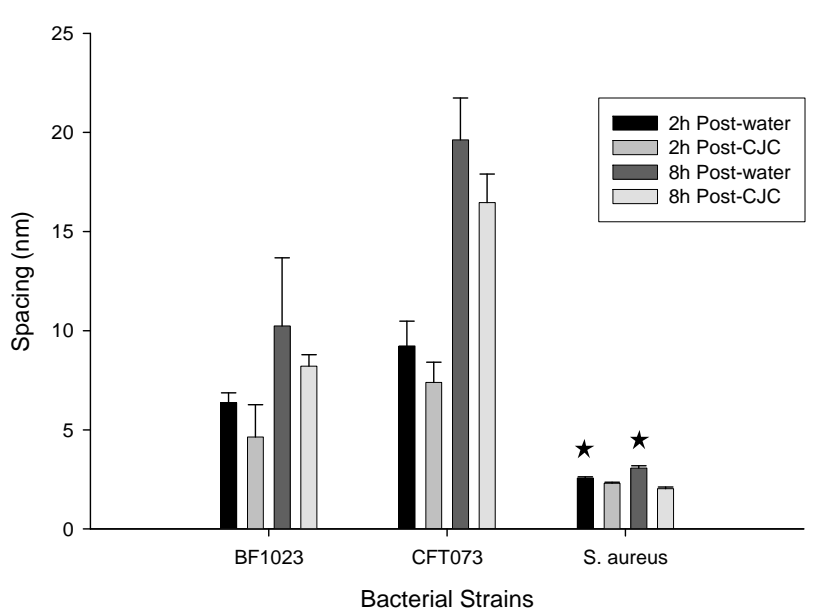

Figure 4. Average spacing values of $E$. coli BF1023, E. coli CFT073, and $S$. aureus at two time intervals (2 and $8 \mathrm{~h}$ ) after beverage consumption (water or CJC). Error bar indicate the standard error of the mean. 5-point black star indicates a statistically significant difference between two treatments at one time point.

ing for BF1023 (4.6 - $10.2 \mathrm{~nm})$ and CFT073 (7.4 - 19.6 $\mathrm{nm}$ ) (Figure 4). Furthermore, when tested at 2 and $8 \mathrm{hs}$ following beverage consumption, $S$. aureus showed a significant decrease in the spacing between grafting sites in the post-CJC urine group compared to its equivalent value in the post-water urine group (Figure 4). This is an indication that the decrease in the spacing between $S$. aureus polymer brushes as a result of drinking CJC lasted up to 8 hours. However, statistical analysis applied on the average spacing values for both $E$. coli strains revealed that there was no significant difference between the spacing in post-CJC urine and post-water urine for both strains after 2 and $8 \mathrm{~h}$ following beverage consumption.

\section{Conclusion}

Exposure to the urine of a volunteer who consumed CJC imparted changes in bacterial adhesion and in the physicochemical properties of bacterial surface molecules, which were not observed in post-water urine. The changes were also dependent on properties of the bacteria. For two E. coli strains, adhesion forces were decreased in post-CJC urine, as quantified via AFM. However, we did observe statistically significant changes in the conformation of the polymers on the E. coli surface in post-CJC urine, compared to post-water urine. For $S$. aureus, we observed both a decrease in adhesion force in post-CJC urine, and statistically significant changes in the conformation of bacterial surface polymers in postCJC urine. Since the nature of the surface molecules on $E$. coli and $S$. aureus are very different, these results suggest that there are multiple mechanisms by which post-CJC compounds in urine can interact with Gram-negative and Gram-positive bacteria.

\section{Acknowledgements}

We are grateful to Ocean Spray for providing cranberry juice cocktail.

This work was supported in part by grants from the National Center for Complementary and Alternative Medicine (NIH R15 AT003385-01A1), the Cranberry Institute, and the Wisconsin Cranberry Board. Funds for the atomic force microscope used in this work were provided in part by a grant from the National Science Foundation (CBET 0922901).

\section{REFERENCES}

[1] B. Foxman, "The Epidemiology of Urinary Tract Infection," Nature Reviews Urology, Vol. 7, No. 12, 2010, pp. 653-660. doi:10.1038/nrurol.2010.190

[2] J. Avorn, M. Monane, J. H. Gurwitz, R. J. Glynn, I. Choodnovskiy and L. A. Lipsitz, "Reduction of Bacteriuria and Pyuria after Ingestion of Cranberry Juice," JAMA, Vol. 271, No. 10, 1994, pp. 751-754. doi:10.1001/jama.1994.03510340041031

[3] M. E. McMurdo, L. Y. Bissett, R. J. Price, G. Phillips and I. K. Crombie, "Does Ingestion of Cranberry Juice Reduce Symptomatic Urinary Tract Infections in Older People in Hospital? A Double-Blind, Placebo-Controlled Trial," Age and Ageing, Vol. 34, No. 3, 2005, pp. 256-261. doi:10.1093/ageing/afi101

[4] J. Salo, M. Uhari, M. Helminen, M. Korppi, T. Nieminen, T. Pokka and T. Kontiokari, "Cranberry Juice for the Prevention of Recurrences of Urinary Tract Infections in Children: A Randomized Placebo-Controlled Trial," Clinical Infectious Diseases, Vol. 54, 2012, pp. 340-346. doi:10.1093/cid/cir801

[5] L. Stothers, "A Randomized Trial to Evaluate Effectiveness and Cost Effectiveness of Naturopathic Cranberry Products as Prophylaxis against Urinary Tract Infection in Women," Canadian Journal of Urology, Vol. 9, 2002, pp. 1558-1662.

[6] C. Barbosa-Cesnik, M. B. Brown, M. Buxton, L. Zhang, J. De Busscher and B. Foxman, "Cranberry Juice Fails to Prevent Recurrent Urinary Tract Infection: Results from a Randomized Placebo-Controlled Trial," Clinical Infectious Diseases, Vol. 52, No. 1, 2011, pp. 23-30. doi: $10.1093 / \mathrm{cid} / \mathrm{ciq} 073$

[7] A. B. Howell, H. Botto, C. Combescure, A. B. BlancPotard, L. Gausa, T. Matsumoto, P. Tenke, A. Sotto and J. P. Lavigne, "Dosage Effect on Uropathogenic Escherichia Coli Anti-Adhesion Activity in Urine Following Consumption of Cranberry Powder Standardized for Proanthocyanidin Content: A Multicentric Randomized Double Blind Study," BMC Infectious Diseases, Vol. 10, 2010, p. 94. doi:10.1186/1471-2334-10-94

[8] T. A. Russo and J. R. Johnson, "Medical and Economic Impact of Extraintestinal Infections Due to Escherichia 
Coli: Focus on an Increasingly Important Endemic Problem," Microbes and Infection, Vol. 5, No. 5, 2003, pp. 449-456. doi:10.1016/S1286-4579(03)00049-2

[9] M. Vittori, A. D’Addessi, F. Sasso, E. Sacco, A. Totaro, A. Calarco, D. D'Agostino, G. Palermo and P. F. Bassi, "Microbiological Follow-Up of Nosocomial Infections in a Single Urological Center," Urologia, 2012.

[10] F. Alvarez-Lerma, M. P. Gracia-Arnillas, M. Palomar, P. Olaechea, J. Insausti, M. J. López-Pueyo, J. J. Otal, R. Gimeno and I. Seijas, "G.d.I.d.E.N.d.V.d.I.N.e. UCI, Urethral Catheter-Related Urinary Infection in Critical Patients Admitted to the ICU," Descriptive Data of the ENVIN-UCI STUDY, Med Intensiva, 2012.

[11] M. Bitsori, S. Maraki, S. Koukouraki and E. Galanakis, "Pseudomonas Aeruginosa Urinary Tract Infection in Children: Risk Factors and Outcomes," The Journal of Urology, Vol. 187, No. 1, 2012, pp. 260-264. doi:10.1016/j.juro.2011.09.035

[12] C. R. Arciola, L. Baldassarri and L. Montanaro, "Presence of icaA and icaD Genes and Slime Production in a Collection of Staphylococcal Strains from Catheter-Associated Infections," Journal of Clinical Microbioloy, Vol. 39, No. 6, 2001, pp. 2151-2156. doi:10.1128/JCM.39.6.2151-2156.2001

[13] C. R. Arciola, D. Campoccia, P. Speziale, L. Montanaro and J. W. Costerton, "Biofilm Formation in Staphylococcus Implant Infections. A Review of Molecular Mechanisms and Implications for Biofilm-Resistant Materials," Biomaterials, Vol. 33, No. 26, 2012, pp. 5967-5982. doi:10.1016/j.biomaterials.2012.05.031

[14] P. A. Pinzón-Arango, Y. Liu and T. A. Camesano, "Role of Cranberry on Bacterial Adhesion Forces and Implications for Escherichia coli-Uroepithelial Cell Attachment," Journal of Medicinal Food, Vol. 12, No. 2, 2009, pp. 259270. doi:10.1089/jmf.2008.0196

[15] A. E. Sobota, "Inhibition of Bacterial Adherence by Cranberry Juice: Potential Use for the Treatment of Urinary Tract Infections," Journal of Urology, Vol. 131, 1984, pp. 1013-1016.

[16] A. B. Howell and B. Foxman, "Cranberry Juice and Adhesion of Antibiotic-Resistant Uropathogens," JAMA, Vol. 287, 2002, pp. 3082-3083. doi:10.1001/jama.287.23.3082

[17] Y. Liu, M. A. Black, L. Caron and T. A. Camesano, "Role of Cranberry Juice on Molecular-Scale Surface Characteristics and Adhesion Behavior of Escherichia coli," Biotechnology and Bioengineering, Vol. 93, No. 2, 2006, pp. 297-305. doi:10.1002/bit.20675

[18] Y. Tao, P. A. Pinzon-Arango, A. B. Howell and T. A. Camesano, "Oral Consumption of Cranberry Juice Cocktail Inhibits Molecular-Scale Adhesion of Uropathogenic Escherichia coli," Journal of Medicinal Food, Vol. 14, No. 7-8, 2011, pp. 739-745.

[19] P. Di Martino, R. Agniel, J. L. Gaillard and P. Denysv, "Effects of Cranberry Juice on Uropathogenic Escherichia coli in Vitro Biofilm Formation," Journal of Chemotherapy, Vol. 17, No. 5, 2005, pp. 563-565.

[20] P. Di Martino, R. Agniel, K. David, C. Templer, J. L.
Gaillard, P. Denys and H. Botto, "Reduction of Escherichia coli Adherence to Uroepithelial Bladder Cells After Consumption of Cranberry Juice: A Double-Blind Randomized Placebo-Controlled Cross-Over Trial," World Journal of Urology, Vol. 24, No. 1, 2006, pp. 21-27. doi:10.1007/s00345-005-0045-Z

[21] V. Dupres, F. D. Menozzi, C. Locht, B. H. Clare, N. L. Abbott, S. Cuenot, C. Bompard, D. Raze and Y. F. Dufrêne, "Nanoscale Mapping and Functional Analysis of Individual Adhesins on Living Bacteria," Nature Methods, Vol. 2, 2005, pp. 515-520. doi:10.1038/nmeth769

[22] D. Alsteens, E. Dague, C. Verbelen, G. Andre, V. Dupres and Y. F. Dufrêne, "Nanoscale Imaging of Microbial Pathogens Using Atomic Force Microscopy," Wiley Interdisciplinary Reviews: Nanomedicine and Nanobiotechnology, Vol. 1, No. 2, 2009, pp. 168-180. doi:10.1002/wnan.18

[23] Y. F. Dufrene, "Atomic Force Microscopy of Fungal Cell Walls: An Update,” Yeast, Vol. 27, No. 8, 2010, pp. 465471. doi:10.1002/yea.1773

[24] I. E. Ivanov, E. N. Kintz, L. A. Porter, J. B. Goldberg, N. A. Burnham and T. A. Camesano, "Relating the Physical Properties of Pseudomonas Aeruginosa Lipopolysaccharides to Virulence Using Atomic Force Microscopy," Journal of Bacteriology, Vol. 193, No. 5, 2011, pp. 12591266. doi:10.1128/JB.01308-10

[25] H.-J. Butt, M. Kappl, H. Mueller and R. Raiteri, "Steric Forces Measured with the Atomic Force Microscope at Various Temperatures," Langmuir, Vol. 15, No. 7, 1999, pp. 2559-2565. doi:10.1021/la981503+

[26] T. A. Camesano and B. E. Logan, "Probing Bacterial Electrosteric Interactions Using Atomic Force Microscopy," Environmental Science and Technology, Vol. 34, No. 16, 2000, pp. 3354-3362. doi:10.1021/es9913176

[27] T. A. Camesano, M. J. Natan and B. E. Logan, “Observation of Changes in Bacterial Cell Morphology Using Tapping Mode Atomic Force Microscopy," Langmuir, Vol. 16, No. 10, 2000, pp. 4563-4572.

[28] P. G. de Gennes, "Polymers at an Interface: A Simplified View," Advances in Colloid and Interface Science, Vol. 27, No. 3-4, 1987, pp. 189-209. doi:10.1016/0001-8686(87)85003-0

[29] S. Alexander, "Adsorption of Chain Molecules with a Polar Head a Scaling Description," Journal of Physics II, Vol. 38, 1977, pp. 983-987.

[30] D. P. Chang, N. I. Abu-Lail, F. Guilak, G. D. Jay and S. Zauscher, "Conformational Mechanics, Adsorption, and Normal Force Interactions of Lubricin and Hyaluronic Acid on Model Surfaces," Langmuir, Vol. 24, No. 4, 2008, pp. 1183-1193. doi:10.1021/la702366t

[31] T. J. Foster and M. Hook, "Surface Protein Adhesins of Staphylococcus Aureus," Trends in Microbiology, Vol. 6, 1998, pp. 484-488. doi:10.1016/S0966-842X(98)01400-0

[32] K. L. Laplante, S. A. Sarkisian, S. Woodmansee, D. C. Rowley and N. P. Seeram, "Effects of Cranberry Extracts on Growth and Biofilm Production of Escherichia coli and Staphylococcus Species," Phytotherapy Research, Vol. 26, No. 9, 2012, pp. 1371-1374. doi:10.1002/ptr.4592 